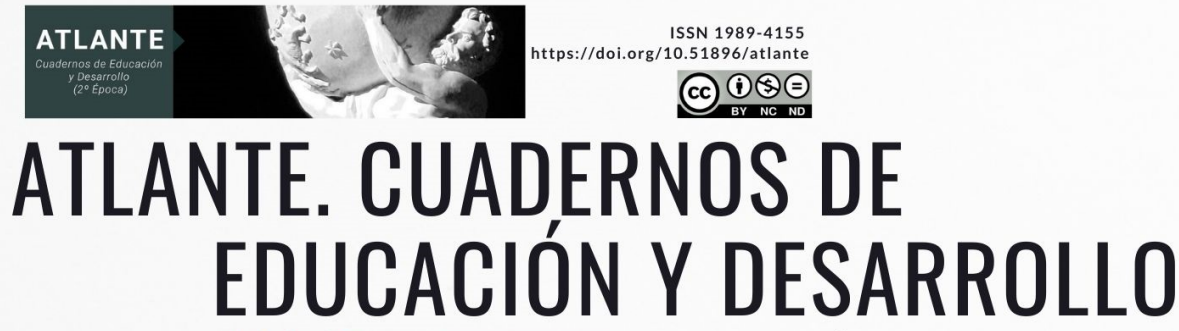

latindex @idEAs EconPapers oDialnet mIAR ÍnDICEs

\title{
NIVEL DE CONGRUENCIAS ESTRATÉGICAS EN LA EDUCACIÓN MEDIA SUPERIOR CON LOS ESTÁNDARES LA OCDE
}

\author{
Orlando Jacinto Itza Gómez Briones \\ académico adscrito a la Universidad de Guadalajara \\ orlando.gomez5418@academicos.udg.mx \\ Alejandro Rocha Galindo \\ académico adscrito a la Universidad de Guadalajara
}

Alejandro.rocha@sems.udg.mx, Alejandro.rocha9375@academicos.udg.mx

Para citar este artículo puede utilizar el siguiente formato:

Orlando Jacinto Itza Gómez Briones y Alejandro Rocha Galindo: "Nivel de congruencias estratégicas en la educación media superior con los estándares la OCDE", Revista Atlante: Cuadernos de Educación y Desarrollo, ISSN: 1989-4155 (vol 13, № 7 octubre-diciembre 2021, pp. 163-168). En línea:

https://doi.org/10.51896/atlante/ZOES2756

\section{RESUMEN}

La educación se define como un medio en el cual el ser humano adquiere conocimientos y valores, con el fin de formar una sociedad competente. Por lo que cada estudiante tiene derecho a recibir una educación suficientemente capaz para el desarrollo de cada individuo. Actualmente la educación media superior sufre un gran rezago educativo, ya que los estudiantes que terminan de esta etapa educativa, egresan sin la preparación necesaria que deberían de tener y el problema se extiende a todo México, según la información obtenida por la OCDE. Esta investigación se llevará a cabo bajo una metodología deductiva, ya que es la ideal para llegar al objetivo el cual consta en comprobar si existe alguna problemática en el proceso educativo en la etapa de educación media superior. Por lo tanto, las autoridades educativas tienen un gran reto, debido a que esta etapa educativa es evaluada por los estándares más altos que maneja la OCDE, por lo que se tiene que crear nuevas estrategias para generar mejores las estrategias educativas y formar mejores alumnos en esta etapa.

Palabras clave: La educación, educación media superior, egresan, problemática, proceso educativo.

\section{LEVEL OF STRATEGY CONGRUENCES IN UPPER SECONDARY WITH OECD STANDARDS}

\section{ABSTRACT}

Education is defined as a means in which the human being acquires knowledge and values, in order to form a competent society. So every student has the right to receive an education sufficiently capable for the development of each individual. Currently the upper secondary education suffers a 
great educational lag, since the students who finish this educational stage, graduate without the necessary preparation that they should have and the problem extends to all of Mexico, according to the information obtained by the OECD. This research will be carried out under a deductive methodology, since it is the ideal one to reach the objective which consists of checking if there is any problem in the educational process in the stage of upper secondary education. Therefore, the educational authorities have a great challenge, because this educational stage is evaluated by the highest standards managed by the OECD, so new strategies have to be created to generate better educational strategies and train better students at this stage.

Keywords: Education, upper secondary education, graduation, problematic, educational process.

\section{INTRODUCCIÓN}

En México la educación se "basará en el respeto irrestricto de la dignidad de las personas, con un enfoque de derechos humanos y de igualdad sustantiva." (MEXICANOS, 2021) .Por lo que cada ciudadano mexicano debe de tener una educación adecuada para cada habitante de México, ya que debe de tener un desarrollo social completo, tanto en conocimiento como en humanismo para así llegar a ser un ciudadano competente para este mundo globalizado y sobre todo apostar en la innovación educativa en todos sus aspectos, debido a que la educación puede tomarse como el motor de cada sociedad, ya que de ahí saldrán personajes, que ayudarán a la evolución de esta mism sociedad; consecuentemente de esto se necesita que a este motor le funcionan todos los engranes, para que de ahí se genere una sociedad competitivamente eficiente para el futuro.

Dentro de esta misma nación se encuentra una educación obligatoria estipulada en la normatividad mexicana, la cual va del pre escolar hasta la educación media superior; hasta el 2017 existían "30, 909, 211" (Educación, 2017) alumnos de los cuales "5, 128, 518" (Educación, 2017) son de educación media superior; esta es la última parte de la educación obligatoria en México, por lo que en esta etapa concluyente, deberían de salir con un alto rendimiento escolar. Esta misma tiene sus inicios en la Nueva España, la cual ha cambiado durante el tiempo con distintos nombres, pero con la misma esencia "En el periodo colonial surgieron los primeros antecedentes de un nivel intermedio entre la educación elemental y la educación superior. En 1551 se estableció la Real y Pontificia Universidad de México, como instituciones educativas preparatorias para las licenciaturas existentes." (México, 2013), siendo un precedente muy fuerte de que esta etapa escolar resulta ser elemental y necesaria para el desarrollo de un individuo y más en la actualidad, ya que en esta etapa educativa deben de egresar los estudiantes competentes para que así ellos puedan saber elegir alguna carrera profesional o en algunos de los casos ser más competentes para ser parte de un trabajo u oficio.

Por lo antes dicho esta etapa de la educación tiene como secuencia ser elemental para el crecimiento intelectual de una personas; la cual tiene como objetivo el "Brindar una educación media superior de calidad, que sea pertinente, relevante, eficaz, eficiente, equitativa y que tenga un alto impacto en la sociedad." (SEMS, 2007) así establecido por la Secretaría de Educación Pública, siendo la institución en México encargada del desarrollo intelectual y humano en México, además se 
debe destacar que la educación es uno de los pilares de cada sociedad debido a que de ahí emanan los futuros profesionistas que van a cambiar o a guiar las siguientes sociedades.

México es uno de los países más reconocidos a nivel mundial por destacar en distintos ámbitos, como en su cultura, por personas extraordinarias, empleos, deportes, etcétera. siendo esto nos traslada a estar en el ojo del mundo al ser protagonistas en distintas cosas como en investigaciones.

Una investigación sobre la competitividad de México nombrado como "México: Sueños sin Oprtunidad" (Insituto Mexicano para la Competitividad A.C., 2019) elaborado en año 2019, en donde México se sitúa en el lugar 34 de 43 países, en la cual señala enormes brechas que nos llevaron a estar en ese lugar; una de esas brechas es la cuestión educativa e indica que "En México, los jóvenes se gradúan de la preparatoria con conocimientos de primero de secundaria." (Instituto Mexicano para la Competitividad A.C., 2019), en consecuencia los estudiantes llegan a egresar a un mundo, con un nivel de competencia bajo y los lleva a no poderse desarrollar completamente dentro de su entorno, sobre todo si este mundo es profesional, creando una nueva brecha en cuestiones laborales al no estar lo suficiente preparados.

Este mismo estudio revela ciertas gráficas, para ser más específicos las GRÁFICA 4, en la página 48 del libro sobre este estudio, está misa señala los diferentes tipos de bachilleratos y en la cual coloca los más prósperos dentro de su estudio, y la cual sitúa lo siguiente y la cual dentro de esta investigación será nombrada como':

\section{figura 1}

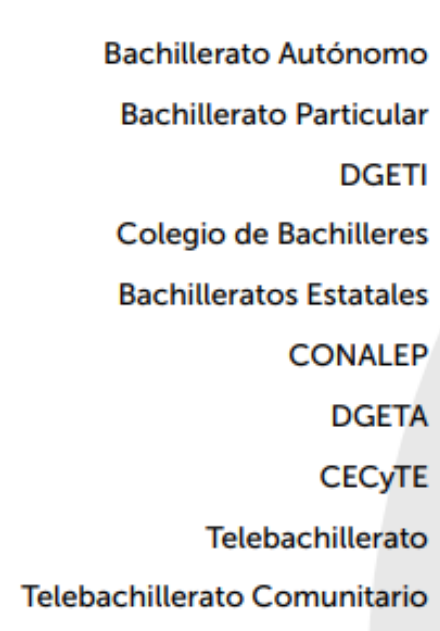

Nivel 1 (menor logro)

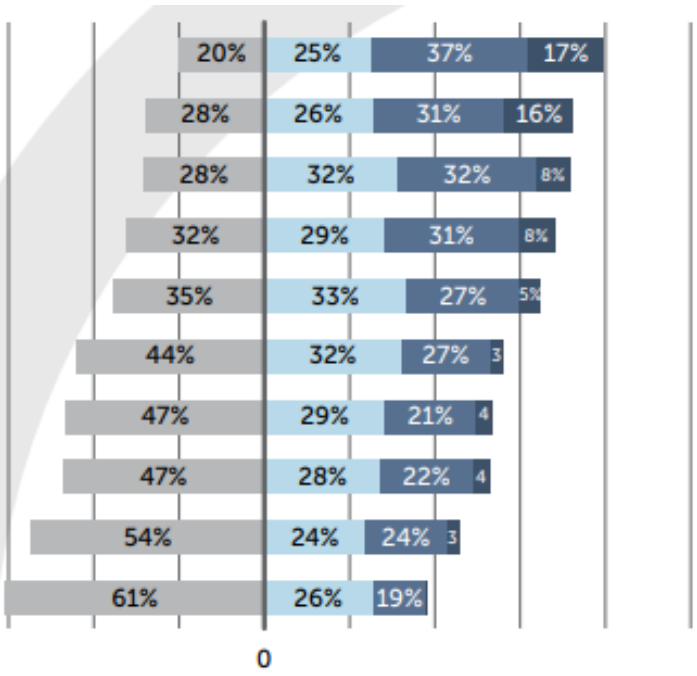

Nivel $2 \square$ Nivel 3
Nivel 4 (mayor logro)

Indice de competitividad internacional 2019

https://www.oecd.org/education/skills-beyond-school/EAG2017CN-Mexico-Spanish.pdf 
Bajo este aspecto, los Bachilleratos Autónomos, son los que se encuentran con más prosperidad en este estudio de competitividad de México y los cuales son alrededor de "545" (Educación, 2017) planteles, siendo esto un factor importante dado a que los bachilleratos restantes son 11,723, de lo cual hace crecer la brecha educativa, debido a que es un porcentaje menor el bachillerato que más posibilidades tiene de prosperidad educativa.

En tales circunstancias La Organización para la Cooperación y el Desarrollo Económicos (OCDE) cuyo objetivo es coordinar políticas económicas y políticas sociales, para promover la prosperidad, la igualdad, tal organización igualmente elaboró una investigación, denominada como "Panorama de la Educación 2017" (OCDE, 2017) en la cual determina que "México tiene una de las mayores proporciones de estudiantes que ingresan a la educación superior en el campo de la ciencia entre los países miembros de la OCDE. " (OCDE, 2017), resultan ser palabras muy rescatables, para la educación en México y sobre todo en la educación media superior que es el cuerpo de esta investigación, ya que los alumnos eligen las áreas con más prosperidad para el futuro, llevando a los estudiantes a un nivel de intelectualidad más alto.

\section{METODOLOGÍA}

El método deductivo resulta ser ideal para este artículo, puesto de que esta investigación necesita premisas que se obtengan de información en común, con los mismos objetivos y de ahí conjuntar y conformar una conclusión en una sola premisa, que tenga la suficiente firmeza en fundamentos para que así puedan generarse distintas soluciones prácticas o teóricas positivas, para el desarrollo estudiantil de los alumnos de educación media superior y así poder formar ciudadanos competentes dentros de sus sociedades.

\section{RESULTADOS Y DISCUSIÓN}

Conjuntado la investigación de "México: Sueños sin Oportunidad" (Instituto Mexicano para la Competitividad A.C., 2019) con la investigación "Panorama de la educación 2017" (OCDE, 2017), nos lleva a un camino con bastantes baches en en camino, pero con posibilidad de recorrerlo puesto que existen evidencias de deficiencias educativas, pero con posibilidades de rescatar a la educación, en vista de que los Bachilleratos autónomos representan una eficiente prosperidad educativa; con las disyuntiva de que estos mismos son minoría en comparación con los bachilleratos restantes de todo Mèxico. Por lo que nos transporta a tomarlos como punto de partida ha tomarlos como base para generar distintas estrategias educativas y enlazar la elección de los alumnos de escoger ciencias y relacionar estas elecciones con las estrategias de lo Bachilleratos autónomos y hacer que las autoridades educativas generen un mejor sistema educativo en la educación media superior.

\section{CONCLUSIONES}

Para concluir en México existe una brecha educativa, sobre todo en educación media superior al momento de egresar y más aún que es el nivel en donde llegan a su fin educativo la mayoría de los ciudadanos en México, pero se tienen grandes posibilidades de salir de este obstáculo y al combinar la elección del área que escogen los alumnos y las estrategias de los Bachilleratos Autónomos; las 
investigaciones "México: Sueños sin Oportunidad" (Instituto Mexicano para la Competitividad A.C., 2019) y "Panorama de la Educación 2017" (OCDE, 2017) unificando los presentes estudios, nos transfiere a aprovechar lo positivo de lo negativo, ya que ser parte de los bachilleratos autónomos nos conduce a que tienen un nivel suficiente educativo, que nos conduce a un relativo éxito y partiendo de este precepto es importante conjuntar el área de que los alumnos que más han escogido y hacer posible la mezcla de estas áreas elegidas a través de los alumnos, con las áreas de menor rendimiento, con la pretensión de los organismos encargados de los educativo y sobre todos los encargados en la educación media superior generen nuevas estrategias para elevar los niveles educativos y mejorar sus desarrollo como estudiantes y ciudadanos al momento de egresar de esta.

\section{BIBLIOGRAFÍA}

Camara de Diputados, H. C. (13 de julio de 1993). Diario Oficial de la Federación. Obtenido de Diario Oficial de la Federación: https://www.sep.gob.mx/work/models/sep1/Resource/558c2c240b12-4676-ad90-8ab78086b184/ley_general_educacion.pdf

Educación, I. N. (2017). Instituto Nacional para la evaluación de la Educación. Obtenido de Instituto Nacional para la evaluación de la Educación: https://www.inee.edu.mx/wpcontent/uploads/2019/01/P2M110.pdf

INEGI. (2020). Información Laboral. Ciudad de Mèxico: INEGI.

Insituto Mexicano para la Competitividad A.C., C. d. (26 de noviembre de 2019). Insituto Mexicano para la Competitividad A.C., Centro de Investigación en Política Pública. Obtenido de Insituto Mexicano para la Competitividad A.C., Centro de Investigación en Política Pública: https://imco.org.mx/indice-de-competitividad-internacional-2019-mexico-suenos-sinoportunidad/

MEXICANOS, C. P. (11 de marzo de 2021). Diario Ofical de la federación. Obtenido de Diario Ofical de la federación: http://www.ordenjuridico.gob.mx/Documentos/Federal/pdf/wo14166.pdf

México, G. d. (14 de octubre de 2013). Gobierno de México. Obtenido de Gobierno de México: http://www.sems.gob.mx/Es_mx/sems/antecedentes_dgb\#: :text=El\%20desarrollo\%20de\% 20la\%20educaci\%C3\%B3n,elemental\%20y\%20la\%20educaci\%C3\%B3n\%20superior.

OCDE. (2017). OCDE. Obtenido de OCDE: https://www.oecd.org/education/skills-beyondschool/EAG2017CN-Mexico-Spanish.pdf

SEMS, P. (10 de agosto de 2007). Secretaria de Educación Publica. Obtenido de Secretaria de Educación Publica: http://www.sep.gob.mx/work/models/sep1/Resource/102426/2.pdf trabajadores, P. d. (1997). Organización Internacional del Trabajo. Obtenido de Organización Internacional del Trabajo: https://www.ilo.org/wcmsp5/groups/public/---ed_protect/--protrav/---safework/documents/normativeinstrument/wcms_112625.pdf 
UNIÓN, C. D. (2 de julio de 2 de julio de 2019). LEY Federal del Trabajo.

http://www.diputados.gob.mx/LeyesBiblio/ref/lft.htm. Obtenido de Honorable Cámara de Diputados: http://www.diputados.gob.mx/LeyesBiblio/ref//ft.htm

UNIÓN, C. D. (5 de julio de 2010). LEY FEDERAL DE PROTECCIÓN DE DATOS PERSONALES EN POSESIÓN DE LOS. http://www.diputados.gob.mx/LeyesBiblio/ref/Ifpdppp.htm. Obtenido de http://www.diputados.gob.mx/LeyesBiblio/ref/lfpdppp.htm 